\title{
Grape Seed Waste Counteracts Aflatoxin B1 Toxicity in Piglet Mesenteric Lymph Nodes
}

\author{
Daniela Eliza Marin*, Cristina Valeria Bulgaru, Cristian Andrei Anghel $\odot$, Gina Cecilia Pistol®, \\ Madalina Ioana Dore, Mihai Laurentiu Palade $[$ and Ionelia Taranu(i)
}

National Institute for Research and Development for Biology and Animal Nutrition, INCDBNA Balotesti, Calea Bucuresti nr 1, Balotesti, 077015 Ilfov, Romania; cristinavaleria11@yahoo.com (C.V.B.); andrei.anghel@ibna.ro (C.A.A.); gina.pistol@ibna.ro (G.C.P.); dore.madalina@ibna.ro (M.I.D.); mihai.palade@ibna.ro (M.L.P.); ionelia.taranu@ibna.ro (I.T.)

* Correspondence: daniela.marin@ibna.ro; Tel.: +40-213512082

Received: 13 November 2020; Accepted: 13 December 2020; Published: 15 December 2020

\begin{abstract}
Aflatoxin B1 (AFB1) is a mycotoxin that frequently contaminates cereals and cereal byproducts. This study investigates the effect of AFB1 on the mesenteric lymph nodes (MLNs) of piglets and evaluates if a diet containing grape seed meal (GSM) can counteract the negative effect of AFB1 on inflammation and oxidative stress. Twenty-four weaned piglets were fed the following diets: Control, AFB1 group (320 $\mathrm{\mu g}$ AFB1/kg feed), GSM group ( $8 \%$ GSM), and AFB1 + GSM group ( $8 \%$ GSM $+320 \mu \mathrm{g}$ AFB1/kg feed) for 30 days. AFB1 has an important antioxidative effect by decreasing the activity of catalase (CAT), superoxide dismutase (SOD), and glutathione peroxidase (GPx) and total antioxidant status. As a result of the exposure to AFB1, an increase of MAP kinases, metalloproteinases, and cytokines, as effectors of an inflammatory response, were observed in the MLNs of intoxicated piglets. GSM induced a reduction of AFB1-induced oxidative stress by increasing the activity of GPx and SOD and by decreasing lipid peroxidation. GSM decreased the inflammatory markers increased by AFB1. These results represent an important and promising way to valorize this waste, which is rich in bioactive compounds, for decreasing AFB1 toxic effects in mesenteric lymph nodes.
\end{abstract}

Keywords: aflatoxin B1; grape seed meal; mesenteric lymph nodes; piglets

Key Contribution: Aflatoxin B1 induces inflammation and oxidative stress in mesenteric lymph nodes. In the mesenteric lymph nodes of AFB1-intoxicated piglets, grape seed meal can improve some markers of inflammation and oxidative stress.

\section{Introduction}

The gut is constantly exposed to potentially harmful contaminants from food or feed, such as mycotoxins [1]. Aflatoxin B1 is a mycotoxin produced by different species of fungi, especially Aspergillus flavus and A. parasiticus [2]. AFB1 is a potent carcinogen in humans and animals [3] and, for this reason, was classified in Group 1 of human carcinogens on the basis of toxicological data. Aflatoxins can contaminate different commodities such as cereals, nuts, dried fruits, and spices [4].

AFB1 is absorbed in the proximal part of the gut at high rates regardless of the species [5]. As the passage of the toxin across the intestinal barrier is very high, AFB1 can compromise the intestinal epithelium even before the absorption in the upper part of the gut. Indeed, it was shown that AFB1 induced inhibition of epithelial cell growth [6], an increase of apoptosis [6], and an increase of apoptotic markers (Bax and caspase-3) [7]. Additionally, AFB1 induced dysregulation of intestinal microbiota [8] and affected intestinal barrier function $[9,10]$. After absorption in the intestinal villi, the lipophilic 
compounds, such as AFB1, are first transported via the mesenteric lymphatic duct and then through the thoracic duct before entering the blood circulation [11]. Mesenteric lymph nodes (MSLs) are important for the proper functioning of the immune system of the gut, acting as filters for nutrients and microbial substances that enter through the lymph in the intestinal lamina propria [12]. MSLs are responsible for tolerance induction to food particles, but they also contribute to the prevention of the systemic inflammatory response that can result from the bypass of portal circulation [13].

AFB1 is a potent immunomodulator as the toxin has been shown to impair both the innate and acquired immune responses [14,15]. However, until now, little is known about the effect of AFB1 on gut immunity and, especially, mesenteric lymph nodes as local effectors of the immune response in the gut.

Grape seed meal (GSM) represents the residue left after grape seed oil extraction; this waste can be recycled in order to enrich the feed rations of farm animals [16,17]. GSM has a high content of bioactive compounds: polyunsaturated fatty acids (mainly linoleic acid, n-6), in addition to oleic acid (n-9), dietary fiber, phenolic acids, resveratrol, proanthocyanidins, and flavonoids [18,19]. In living organisms, the beneficial effects of bioactive plant compounds as counteracting agents for AFB1 toxicity have been investigated both in vivo and in vitro [20]. An in-vitro study performed on human hepatocytes has shown that an extract from palm kernel cake, rich in phenolics, has beneficial effects by upregulating the genes involved in the response to oxidative stress and downregulating the genes associated with inflammation and apoptosis in AFB1-exposed hepatocytes [21]. Additionally, in vivo studies have demonstrated that in rats, oxidized tea polyphenols can form a complex with AFB1 that leads to a decrease of AFB1 absorption and, implicitly, a reduction of its toxicity [22].

In particular, the bioactive compounds from grape seed were shown to have beneficial effects on intoxication with AFB1. In a recent study, the supplementation of an AFB1-contaminated diet with grape seed proanthocyanidin extract reduced AFB1 residue in the liver and significantly mitigated the negative effects caused by AFB1 in broiler chickens [23]. Through their alimentation, rich in cereals, especially maize, pigs are particularly exposed to mycotoxins. We have demonstrated that GSM addition into a diet contaminated with AFB1 ameliorates histological liver injuries, reduces the parameters associated with oxidative stress in piglets [24], and is able to change the microbiota composition already affected by AFB1 exposure [25]. The present study is a continuation of our previous studies and aims to investigate if AFB1 can affect mesenteric lymph nodes as the gate of toxic substances in intestinal lamina propria. Additionally, this study aims to evaluate if the administration of a diet containing grape seed meal can counteract the negative effects of AFB1 on inflammation and oxidative response.

\section{Results}

\subsection{Effect of Grape Seed Meal and Aflatoxin B1 on Oxidative Damage in Mesenteric Lymph Nodes}

AFB1 induces a significative decrease in the activity of the enzymes involved in the response to oxidative stress, with $27 \%$ for catalase (CAT; $p=0.0248), 22 \%$ for glutathione peroxidase (GPx; $p=$ 0.0034 ), and $10.2 \%$ for superoxide dismutase (SOD; $p<0.0001$ ), as compared with the control piglets (Figure 1). Additionally, exposure of piglets to $320 \mu \mathrm{g}$ AFB1/kg feed, significantly decreased the total antioxidant status $(-20.5 \% ; p=0.0001)$ associated with an increase of lipid peroxidation $(+52.3 \% ; p$ $=0.0001$ ), as assessed by the TBARS method. When piglet diet included $8 \%$ of grape seed meal, no effect was observed on the activity of the enzymes involved in antioxidative defense, total antioxidant capacity (TAC), or lipid peroxidation. However, the concomitant administration of both grape seed meal and toxin resulted in a significant increase of GPx (by 119.3\%; $p=0.0048$ ), SOD (by 105.9\%; $p=$ 0.0046 ), and TAC (by 112\%; $p=0.0180$ ), as compared with the AFB1 group, while decreasing lipid peroxidation (by $12.3 \% ; p=0.018)$. GSM was not able to significantly increase CAT activity $(p=0.331$, as compared with the AFB1 group). 
CAT

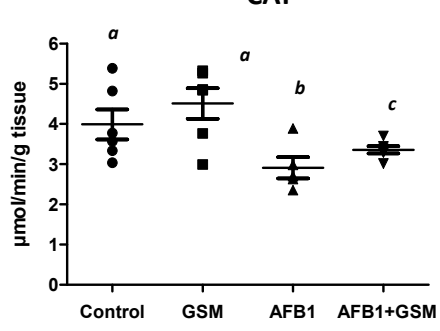

GPX

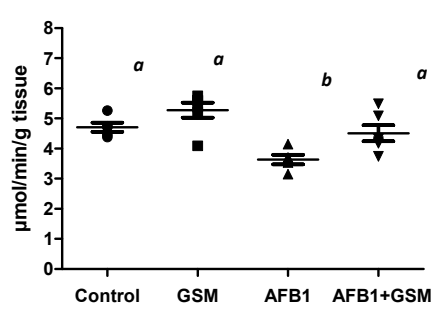

SOD

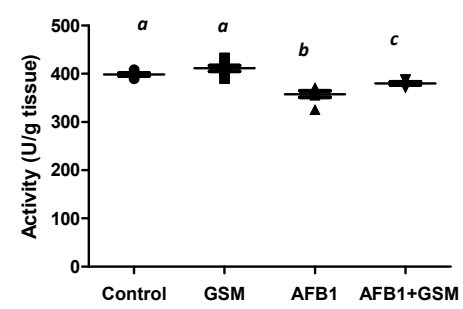

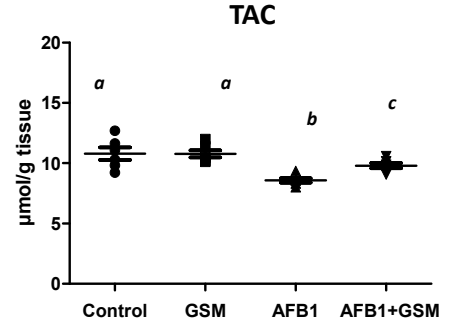

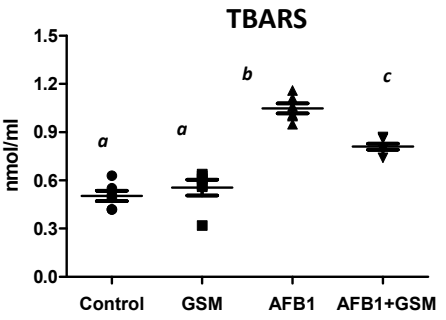

Figure 1. Effect of grape seed meal and AFB1 on oxidative damage in mesenteric lymph nodes. Data represent mean $\pm \mathrm{SD}, n=6$. One-way ANOVA tests, followed by a Fisher's PSLD test, were used for statistical data analyses. ${ }^{\mathrm{a}-\mathrm{c}}$ indicate statistically significant differences between treatments $(p<0.05)$.

2.2. Effect of Grape Seed Meal and Aflatoxin B1 on Inflammatory Cytokine Synthesis in Mesenteric Lymph Nodes

Piglets' exposure to AFB1 contaminated diet was responsible for a a significant increase in the synthesis of IL-1 $\beta(p=0.0068)$, IL-8 $(p=0.0010)$, and for a tendency of increase in the synthesis of IL-6, IFN- $\gamma$, and TNF- $\alpha(p>0.05$; Figure 2).
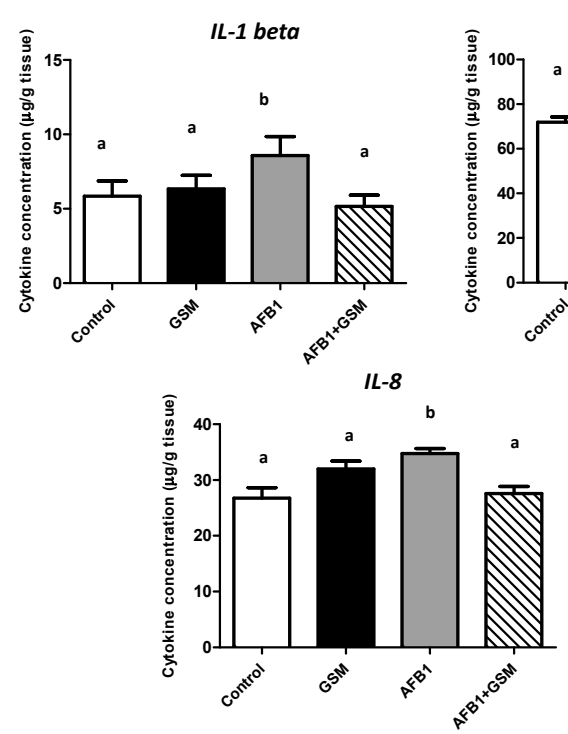
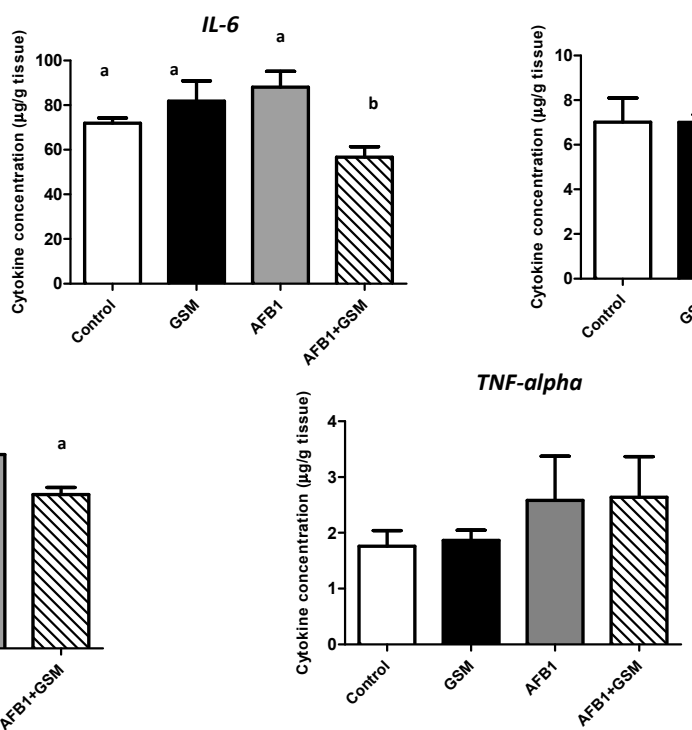

Figure 2. Effect of grape seed meal and AFB1 on inflammatory cytokine synthesis in mesenteric lymph nodes. Data represent means $\pm \mathrm{SD}, n=6$. One-way ANOVA tests, followed by Fisher's PSLD test, were used for statistical data analyses. ${ }^{\mathrm{a}-\mathrm{c}}$ indicate statistically significant differences between treatments $(p$ $<0.05)$.

GSM diet did not affect the synthesis of inflammatory cytokines, the concentration of analyzed cytokines being very similar to the concentration measured in the mesenteric lymph nodes of the control group. 
GSM administration to the piglets exposed to AFB1 (AFB1 + GSM diet) had an important anti-inflammatory effect, decreasing inflammatory cytokine synthesis towards the control level. Inclusion of GSM into the AFB1-contaminated diet (AFB1 + GSM group) was responsible for a decrease in the synthesis of IL-1 $\beta(8.57 \pm 2.97$ to $5.16 \pm 1.75 \mu \mathrm{g} / \mathrm{g}$ tissue $)$, IL-8 $(34.7 \pm 2.07$ to $27.59 \pm 3.07 \mu \mathrm{g} / \mathrm{g}$ tissue), IL-6 (81.9 \pm 14.9 to $57.97 \pm 9.88)$, and IFN- $\gamma(8.13 \pm 2.54$ to $6.72 \pm 1.47 \mu \mathrm{g} / \mathrm{g}$ tissue $)$ towards the levels of the control group.

This decrease was significant for IL-1 $\beta(p=0.0009)$, IL-6 $(p=0.044)$, and IL-8 $(p=0.0018)$ and not for IFN- $\gamma(p=0.371)$. GSM was not able to counteract the increase of TNF- $\alpha$ induced by the toxin $(p=$ $0.389)$.

\subsection{Effect of Grape Seed Meal and Aflatoxin B1 on Cell Signaling Pathways in Mesenteric Lymph Nodes}

In order to validate the qPCR analysis and to demonstrate that GSM can restore the negative effects, such as inflammation and the oxidative response triggered by AFB1, we assessed by Western blot the expression of three proteins involved in NF-kB and Nrf2 signaling pathways: phospho-p38 MAPK, phospho-NF-kBp65, and Nrf2 (Figure 3A-C).

\section{Phospho NF-kB1}

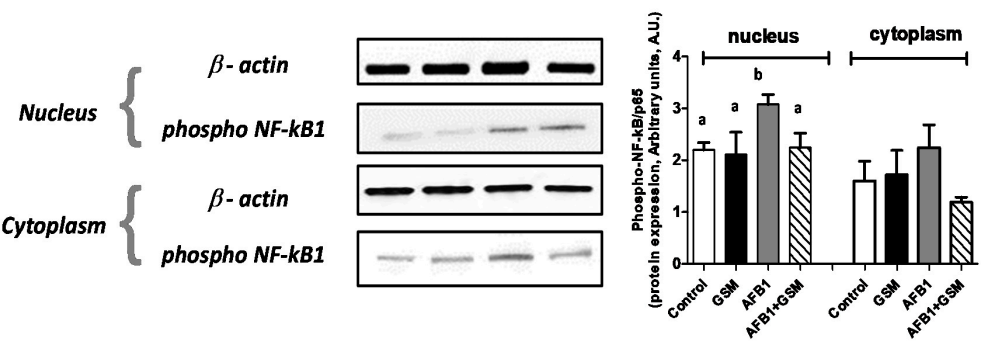

(A)

Phospho p38 MAPK
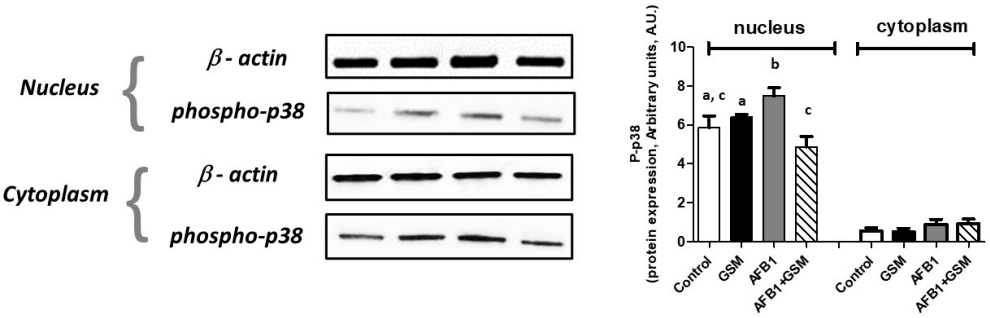

(B)

Nfr2
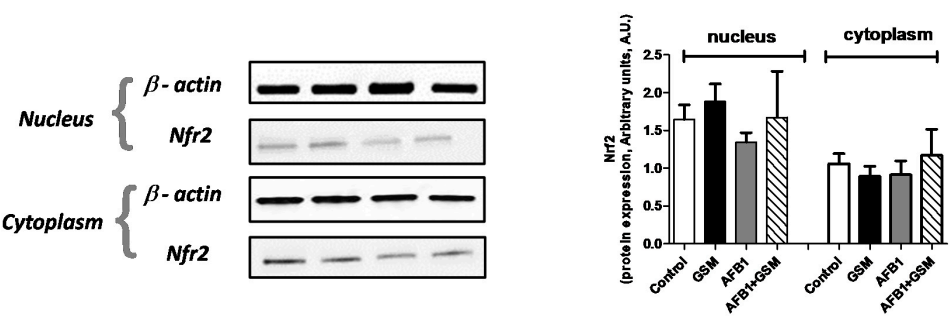

(C)

Figure 3. Effect of grape seed meal and aflatoxin B1 on cell signaling pathways in mesenteric lymph nodes: (A) phosphor-NF-kB1; (B) phosphor-p38 MAPK; (C) Nrf2. Data represent mean \pm SD, $n=$ 6. One-way ANOVA tests, followed by Fisher's PSLD test, were used for statistical data analyses. a-c indicate statistically significant differences between treatments $(p<0.05)$. 
Feeding piglets with the diet contaminated with $320 \mu \mathrm{g}$ AFB1/kg feed resulted in a significant nuclear increase in protein expression of both phosphorylated forms of NF-kBp65 $(p=0.049$, Figure 3A) and p38 MAPK ( $p=0.028$, Figure 3B), while Nrf2 expression was not affected $(p=0.55$, Figure $3 C)$ in the AFB1 nuclear fraction when compared with control.

No significant alteration of the expression of these three proteins was noticed in the cytoplasm, although a slight increase of NF-kB expression in the AFB1 group was observed. As compared with the control, concomitant administration of both GSM and AFB1 resulted in a decrease of the expression of both phospho-p38 MAPK (4.86 \pm 0.54 vs. $5.86 \pm 0.59$ A.U.) and phospho-NF-kBp65 (2.24 \pm 0.28 vs. $2.19 \pm 0.14$ A.U.).

Additionally, the expression of Nrf2 increased toward the control level in the AFB1 + GSM group as compared with the control in both nucleus (1.64 \pm 0.19 vs. $1.67 \pm 0.69$ A.U.) and cytoplasm (1.06 \pm 0.11 vs. $1.17 \pm 0.28$ A.U.). The expression of the proteins involved in the inflammation signaling pathway decreased significantly in the AFB1 + GSM group as compared with the AFB1 group: -35\% for phospho-p38 MAPK ( $p=0.0017)$ and $-27 \%$ for phospho-NF-kBp65 $(p=0.05)$, respectively.

\subsection{Effect of Grape Seed Meal and Aflatoxin B1 on mRNA Gene Expression Involved in Inflammation}

Exposure to AFB1-contaminated diet was responsible for a significant increase in the expression of the majority of the investigated genes. AFB1 contamination led to a highly significant increase in the expression of several proinflammatory markers: IL-8 ( $p=0.001)$, IL-1 $\beta(p=0.023)$, and IL-18 (0.07) (Table 1).

Looking at the gene expression encoding for signaling molecules with a key role in the inflammation pathway, a similar increase was observed for (i) transcription factors: c-Jun $(p=0.013)$, NF-kB p65 ( $p=$ 0.004), STAT3 ( $p=0.011)$; (ii) MAP kinases: extracellular signal-regulated kinase ERK $1(p=0.039)$; (iii) metalloproteinase MMP2 ( $p=0.001)$. When compared with the control diet, GSM alone had no effect on inflammatory markers or signaling molecules, with the exception of some genes, but resulted in a decrease in inflammatory response of the mesenteric lymph nodes, compared with the AFB1 group. In the AFB + GSM group, GSM was responsible for a significant decrease of the expression of ERK-1 ( $p=$ $0.006)$, c-Jun $(p=0.028)$, IL-18 $(p=0.002)$, and IFN- $\gamma(p=0.002)$, while the AFB1 $\times$ GSM effect for the other gene expressions was not significant $(p>0.05)$.

Table 1. Effect of the exposure of piglets to aflatoxin B1 and/or aflatoxin B1 + grape seed meal on selected gene expression in mesenteric lymph nodes.

\begin{tabular}{ccccccc}
\hline Gene & Control & GSM & AFB1 & GSM + & \multicolumn{2}{c}{$p$ Value } \\
\cline { 7 - 7 } Expression & & & & AFB1 & AFB1 Effect & AFB1 $\times$ GSM Effect \\
\hline ERK-1 & $1.0 \pm 0.73$ & $3.53 \pm 0.63$ & $34.6 \pm 0.63$ & $1.96 \pm 0.73$ & 0.039 & 0.006 \\
c-JUN & $1.0 \pm 0.28$ & $0.94 \pm 0.35$ & $6.83 \pm 0.62$ & $1.07 \pm 0.13$ & 0.013 & 0.028 \\
p38 a & $1.0 \pm 0.68$ & $0.86 \pm 0.03$ & $2.21 \pm 0.64$ & $0.47 \pm 0.24$ & 0.108 & 0.782 \\
JNK1 & $1.0 \pm 0.59$ & $0.56 \pm 0.48$ & $2.53 \pm 0.57$ & $0.78 \pm 0.15$ & 0.211 & 0.543 \\
JNK2 & $1.0 \pm 0.28$ & $0.62 \pm 0.51$ & $3.12 \pm 0.69$ & $0.73 \pm 0.25$ & 0.170 & 0.297 \\
NF-kB p65 & $1.0 \pm 0.36$ & $0.80 \pm 0.66$ & $7.97 \pm 0.42$ & $1.57 \pm 0.08$ & 0.004 & 0.112 \\
STAT 3 & $1.0 \pm 0.84$ & $1.25 \pm 0.62$ & $17.4 \pm 0.71$ & $2.93 \pm 0.17$ & 0.011 & 0.681 \\
MMP 2 & $1.0 \pm 0.45$ & $0.75 \pm 0.58$ & $12.5 \pm 0.66$ & $2.34 \pm 0.10$ & 0.001 & 0.168 \\
TNF $\alpha$ & $1.0 \pm 0.10$ & $0.95 \pm 0.05$ & $7.75 \pm 0.49$ & $3.22 \pm 0.07$ & 0.187 & 0.107 \\
IL-1 $\beta$ & $1.0 \pm 0.15$ & $0.76 \pm 0.34$ & $15.3 \pm 0.71$ & $0.66 \pm 0.10$ & 0.023 & 0.073 \\
IL-8 & $1.0 \pm 0.49$ & $0.94 \pm 0.05$ & $17.5 \pm 0.37$ & $0.87 \pm 0.46$ & 0.001 & 0.168 \\
IL-18 & $1.0 \pm 0.28$ & $0.96 \pm 0.61$ & $26.8 \pm 0.22$ & $0.35 \pm 0.14$ & 0.076 & 0.002 \\
IFN $\gamma$ & $1.0 \pm 0.25$ & $1.68 \pm 0.82$ & $14.2 \pm 0.29$ & $0.37 \pm 0.14$ & 0.326 & 0.002 \\
\hline
\end{tabular}

2.5. Correlations between Gene Expressions of Transcription Factors, MAP Kinases, Metalloproteinases, and Cytokines in Pigs Fed AFB1 and AFB1 + GSM Diets

In order to confirm the inflammatory effect of AFB1 and to better understand the mechanism involved in GSM action in counteracting the AFB1 effect, mathematical correlations were established between the expressions of transcription factors, MAP kinases, metalloproteinases, and cytokines in 
mesenteric lymph node samples derived from pigs fed AFB1 or AFB1 + GSM diets. As expected, the expression of all the inflammatory markers analyzed in the present paper was positively correlated (Figure 4).

\begin{tabular}{|c|c|c|c|c|c|c|c|c|c|c|c|c|c|}
\hline & ERK-1 & c-JUN & $p 38 \alpha$ & JNK1 & JNK2 & NF-kB & STAT 3 & MMP 2 & TNF $\alpha$ & IL-1 $\beta$ & IL-8 & IL-18 & IFN $\gamma$ \\
\hline ERK-1 & 1 & 0.929 & 0.541 & 0.832 & 0.789 & 0.927 & 0.512 & 0.871 & 0.666 & 0.317 & 0.651 & 0.873 & 0.577 \\
\hline$c-J U N$ & & 1 & 0.571 & 0.84 & 0.887 & 0.951 & 0.647 & 0.923 & 0.694 & 0.285 & 0.628 & 0.836 & 0.654 \\
\hline p38 $\alpha$ & & & 1 & 0.597 & 0.766 & 0.33 & 0.397 & 0.345 & 0.701 & 0.325 & 0.876 & 0.745 & 0.612 \\
\hline JNK1 & & & & 1 & 0.904 & 0.808 & 0.311 & 0.72 & 0.468 & 0.337 & 0.531 & 0.7 & 0.414 \\
\hline JNK2 & & & & & 1 & 0.792 & 0.506 & 0.765 & 0.595 & 0.188 & 0.691 & 0.798 & 0.541 \\
\hline$N F-k B$ & & & & & & 1 & 0.573 & 0.958 & 0.572 & 0.259 & 0.488 & 0.75 & 0.557 \\
\hline STAT 3 & & & & & & & 1 & 0.662 & 0.496 & 0.041 & 0.586 & 0.641 & 0.78 \\
\hline MMP 2 & & & & & & & & 1 & 0.544 & 0.097 & 0.464 & 0.689 & 0.573 \\
\hline TNF $\alpha$ & & & & & & & & & 1 & 0.606 & 0.834 & 0.894 & 0.85 \\
\hline IL-1 $\beta$ & & & & & & & & & & 1 & 0.37 & 0.569 & 0.573 \\
\hline IL-8 & & & & & & & & & & & 1 & 0.864 & 0.787 \\
\hline IL-18 & & & & & & & & & & & & 1 & 0.89 \\
\hline IFN $\gamma$ & & & & & & & & & & & & & 1 \\
\hline
\end{tabular}

Figure 4. Gene expression correlations in lymph nodes of piglets exposed to AFB1 versus control.

Highly significant correlation was obtained between the expression of NF-kB, the key signaling molecule of the inflammation pathway, and the expression of (i) transcription factor c-Jun $\left(R^{2}=\right.$ 0.95); (ii) MAPK signaling molecules: ERK1 $\left(R^{2}=0.927\right)$, JNK1 $\left(R^{2}=0.808\right)$, JNK2 $\left(R^{2}=0.792\right)$; (iii) metalloproteinase MMP2 $\left(R^{2}=0.958\right)$; (iv) inflammatory cytokine IL-18 $\left(R^{2}=0.75\right)$. When mathematical correlations were performed between inflammatory gene expressions of lymph nodes derived from the AFB1 + GSM group and those of the AFB1 group (Figure 5), a highly significant correlation were observed between the expression of NF-kB and the expression of c-JUN $\left(R^{2}=0.965\right), \mathrm{p} 38\left(R^{2}=\right.$ $0.924)$, JNK $1\left(R^{2}=0.828\right)$, JNK $2\left(R^{2}=0.986\right)$, STAT $3\left(R^{2}=0.982\right)$, MMP $2\left(R^{2}=0.952\right)$, TNF- $\alpha\left(R^{2}\right.$ $=0.817)$, IL-8 $\left(R^{2}=0.822\right)$, IL-18 $\left(R^{2}=0.872\right)$, and IFN- $\gamma\left(R^{2}=0.875\right)$, which clearly demonstrate the anti-inflammatory effect of GSM on the mesenteric lymph nodes of AFB1-intoxicated piglets.

\begin{tabular}{|c|c|c|c|c|c|c|c|c|c|c|c|c|c|}
\hline & ERK-1 & $c-J U N$ & $p 38 \alpha$ & JNK1 & JNK2 & NF-kB & STAT 3 & MMP 2 & TNF $\alpha$ & $I L-1 \beta$ & IL-8 & IL-18 & IFN $\gamma$ \\
\hline ERK-1 & 1 & 0.727 & 0.704 & 0.616 & 0.672 & 0.745 & 0.695 & 0.795 & 0.89 & 0.55 & 0.38 & 0.764 & 0.748 \\
\hline$c-J U N$ & & 1 & 0.87 & 0.799 & 0.863 & 0.965 & 0.953 & 0.936 & 0.786 & 0.417 & 0.81 & 0.876 & 0.824 \\
\hline$p 38 \alpha$ & & & 1 & 0.897 & 0.963 & 0.924 & 0.964 & 0.966 & 0.695 & 0.288 & 0.679 & 0.689 & 0.751 \\
\hline JNK1 & & & & 1 & 0.937 & 0.828 & 0.87 & 0.829 & 0.576 & 0.38 & 0.59 & 0.672 & 0.702 \\
\hline JNK2 & & & & & 1 & 0.896 & 0.927 & 0.929 & 0.623 & 0.261 & 0.672 & 0.664 & 0.7 \\
\hline$N F-k B$ & & & & & & 1 & 0.982 & 0.952 & 0.817 & 0.507 & 0.822 & 0.872 & 0.875 \\
\hline STAT 3 & & & & & & & 1 & 0.966 & 0.755 & 0.436 & 0.801 & 0.8 & 0.835 \\
\hline MMP 2 & & & & & & & & 1 & 0.799 & 0.268 & 0.739 & 0.795 & 0.796 \\
\hline TNF $\alpha$ & & & & & & & & & 1 & 0.529 & 0.615 & 0.876 & 0.907 \\
\hline IL-1 $\beta$ & & & & & & & & & & 1 & 0.518 & 0.593 & 0.727 \\
\hline IL-8 & & & & & & & & & & & 1 & 0.774 & 0.776 \\
\hline IL-18 & & & & & & & & & & & & 1 & 0.898 \\
\hline IFN $\gamma$ & & & & & & & & & & & & & 1 \\
\hline
\end{tabular}

Figure 5. Gene expression correlations in lymph nodes of piglets exposed to AFB1+ GSM versus AFB1-exposed piglets.

\section{Discussion}

Mycotoxins are responsible for important decreases in productivity and health in the animal sector, and finding new solutions for the reduction of their toxic effects represent a continuous challenge for scientists. For some mycotoxins, such as aflatoxins, zearalenone, and ochratoxin, the use of high-affinity binders represents a promising solution as they can form stable complexes that cannot be adsorbed in the gut and are eliminated through feces, thus reducing the toxins' systemic absorption [26,27]. 
According to Commission Regulation (EC) no 386/2009, mycotoxin adsorbents, which are able to mitigate the toxins' effects through the reduction of intestinal absorption, were classified as a new functional group of feed additives. As stipulated in this regulation, the mycotoxin adsorbents should suppress or reduce the absorption, promote the excretion of mycotoxins and, at the same time, increase the quality of the feed, assuring the protection of public and animal health. At present, the use of mycotoxin adsorbents represents the best method for protecting animals against the harmful effects of contaminated feed $[28,29]$.

From the mycotoxin adsorbents group, aluminosilicates are the most tested, but their use is limited due to their capacity to bind other molecules besides mycotoxins, such as vitamins and minerals that cannot be further absorbed in the gut [30]. Additionally, many binders have proven a high efficacy for mycotoxin adsorption using in-vitro abiotic systems, but these qualities were not confirmed by the in vivo studies [31].

Grape residues, as agroindustrial waste, are frequently discarded in open areas, representing a problem for the environment [32]. In order to avoid this, investigations have been done for their use for other purposes. Highlighting their chemical composition, rich in bioactive compounds, has led to their use as food/feed additives and pharmaceuticals [33]. For example, grape seed waste has been used in animal farm production in order to get functional foods, such as eggs or meat enriched in PUFAs or polyphenols [16,34].

The use of grape waste as binders of mycotoxins is of recent interest. Thus, the bioactive compounds from grape byproducts (grape pomace) were shown to efficiently adsorb AFB1 in abiotic systems [35]. Grape pomace was also efficient in reducing the urinary mycotoxin biomarker of AFB1 and zearalenone in an in-vivo trial, where piglets were fed a bolus contaminated with a mixture of mycotoxins (fumonisin B1, deoxynivalenol, zearalenone, AFB1, and ochratoxin A), through the reduction of gastrointestinal mycotoxin absorption [36].

As the information related to the effect of AFB1 on the lymphatic system and, in particular, the lymph nodes are scarce, the present study investigated the effect of AFB1 on the mesenteric lymph nodes of piglets after weaning. Additionally, we have investigated if a diet containing grape seed meal can counteract the negative effect of AFB1 on inflammation and oxidative response in piglets' MSL.

Many studies have shown that oxidative stress represents one of the causes for AFB1-induced toxicity, which leads to the generation of reactive oxygen species (ROS), resulting in lipid, protein, and DNA damage and, consequently, cell injury [37].

Oxidative stress plays a negative role in chronic inflammatory diseases [38], and our previous data indicate that oxidative stress and inflammation are tightly correlated in animals exposed to mycotoxins $[39,40]$.

In the present study, our results have shown that AFB1 has an important antioxidative effect by decreasing the activity of principal enzymes involved in the response to oxidative stress (CAT, GPx, SOD) and total antioxidant status while increasing lipid peroxidation, as compared to oxidative stress parameters in the MSL of control piglets. As a result of the exposure to AFB1, an increase of transcription factors, MAP kinases as signaling molecules, and metalloproteinases and cytokines as effectors of inflammatory response were observed in the mesenteric lymph nodes of the intoxicated piglets. According to the most recent data, AFB1 has a rather biphasic effect on the immune response, with a stimulatory effect in the first phase, followed by a suppressive action in the second phase [41]. Immuno-stimulation induced by AFB1 increases the synthesis of inflammatory markers and free radicals, which lead to chronic inflammation and cancer [42,43]. Anti-inflammatory activities of plant bioactive compounds have been reported in acute and chronic inflammation in animal models [44,45], and recent data have shown that they can be used for counteracting the toxic effect of AFB1 [24,46,47]. Our previous results have shown that GSM addition into AFB1-contaminated diet induced the amelioration of liver injuries and decreased inflammation and oxidative stress in the liver of intoxicated piglets by decreasing the MAPKs and NF- $\mathrm{KB}$ signaling pathways overexpressed by the AFB1 diet [24]. 
Although the immune system represents one of AFB1's targets, few studies have investigated the potential beneficial role of bioactive compounds on the immune organs. For example, curcumin, a powerful plant antioxidant, decreases the weight of spleen and bursa of Fabricius, as well as the ratio of spleen/bursa, in broilers fed AFB1, towards similar values to the control group [48]. Additionally, in AFB1-intoxicated mice, lycopene, another plant bioactive compound, can alleviate AFB1-induced immunosuppression by increasing spleen weight, spleen coefficient, T-lymphocyte subsets, and IL-2, IFN- $\gamma$, and TNF- $\alpha$ gene expression in spleen [49]. Similarly, our results have shown that GSM was able to decrease proinflammatory cytokine gene expression and protein synthesis (IL-1 $\beta$, IL-6, IL-8, IL-18, and IFN- $\gamma$ ), as well as the gene and protein expressions of important markers of signaling inflammation-ERK-1, c-Jun, phospho-p38 MAPK, phospho-NF-kBp65-as molecules involved in the NF-kB signaling pathway.

It was shown that phytocompounds can alleviate the immunosuppression induced by AFB1 in the spleen of mice through the inhibition of oxidative stress and mitochondria-mediated apoptosis [49]. Indeed, our results have shown that GSM induced a reduction of AFB1-induced oxidative stress by increasing the activity of enzymes involved in the response to oxidative stress (GPx and SOD) and decreasing lipid peroxidation.

The mechanism responsible for the beneficial effect of GSM in counteracting AFB1 toxicity is not clear. Recent studies have shown that grape pomace was able to efficiently bind AFB1, decreasing in this way the toxin's absorption and increasing its excretion $[35,36]$. Likewise, our in vitro studies, performed on a panel of eight agroindustrial wastes, have shown that GSM was the most efficient binder of AFB1; these results suggest that this waste has a high capacity to bind AFB1 (Palade et al., submitted). The decrease of the toxic effects of AFB1 in the MSL of the AFB1 + GSM group, observed in our study, can be related to the capacity of GSM to adsorb AFB1 and increase its elimination.

Some hypotheses concerning the GSM way of action can be formulated based on previous literature studies. The first one, related to the high cellulose content (37.8\%) of GSM [24], has a great potential to adsorb AFB1 by electrostatic attractions and hydrogen bonding, resulting in the formation of a mycotoxin monolayer on its surface [48]. The second one is based on the ability of polyphenols to form a complex with AFB1 mycotoxins. In a recent study, Lu et al. (2017) demonstrated that polyphenols from fermented tea can reduce AFB1-induced liver injury as they bind the toxin in a complex (C-AFB1 complex) and, consequently, inhibit AFB1 absorption and increase toxin elimination through feces [22]. Another interesting hypothesis is that of Ali Rajput et al., who considered that the protective effects of the bioactive compound proanthocyanidin, from grape seed, may be due to AFB1 biotransformation in the gut, which leads to the reduction of AFB1 absorption [23]. However, more studies are necessary to elucidate the interaction between grape waste and AFB1.

\section{Conclusions}

In conclusion, our study demonstrates that before being transported via the mesenteric lymphatic duct and entering the blood circulation, AFB1 exerts proinflammatory and pro-oxidative effects in the mesenteric lymph nodes of intoxicated piglets. Grape seed meal, a waste product generated after oil extraction, has been shown to have the capacity to reduce the inflammation and oxidative stress triggered by AFB1. These results represent an important and promising way to valorize this waste, rich in bioactive compounds, for decreasing AFB1 toxic effects on mesenteric lymph nodes.

\section{Materials and Methods}

\subsection{Animals and Dietary Treatments}

Twenty-four crossbred weaned piglets (TOPIGS-40), 4 weeks old, were randomly assigned to the experimental groups and fed the following treatments for 30 days: Control (fed a maize-soybean diet), AFB1 group (diet contaminated with $320 \mu \mathrm{g}$ AFB1/kg feed), GSM group (diet with $8 \%$ grape seed meal included), and AFB1 + GSM group (basal diet with $8 \%$ GSM and $320 \mu \mathrm{g}$ AFB1/kg feed), as previously 
described by Taranu et al. [50]. Each experimental group was represented by 6 piglets ( 2 pens/treatment and 3 pigs/pen). Piglets had access ad libitum to water and feed during the experimental period.

The experimental protocol was approved on 5 February 2020 by the Ethical Committee (no. 52/2014 of INCDBNA Balotesti) and the animal handling was done in accordance with EU Council Directive 98/58/EC and Romanian Law 206/2004.

Animals were slaughtered at the end of the experiment by exsanguination, and samples of the mesenteric lymph nodes were taken on ice and stored at $-80^{\circ} \mathrm{C}$ until the assessment of the immune and stress oxidative parameters.

\subsection{Composition of the Grape Seed Meal}

Dried grape seed meal (GSM), resulted after oil extraction, was provided by S.C. OLEOMET-S.R.L., Bucharest, Romania. Analyses consisting of chemical composition (fat, protein, ash, fibers), total and specific polyphenol content, as well as polyunsaturated fatty acids (PUFA) and antioxidant capacity, were performed for GSM characterization, as already described by Taranu et al. [51].

\subsection{Toxin}

Pure AFB1 (FERMENTEC, Jerusalem, Israel) was used to contaminate both AFB1 and GSM + AFB1 diets. Briefly, $50 \mathrm{mg}$ toxin was dissolved in dimethyl sulfoxide and mixed with the control diet in order to achieve a final AFB1 concentration of $320 \mu \mathrm{g} / \mathrm{kg}$ diet. The final AFB1 concentration was confirmed by ELISA and UPLC analysis $(320 \pm 10.9 \mu \mathrm{g}$ AFB1 $/ \mathrm{kg}$ diet $)$. In the control diet, the AFB1 concentration was $2.4 \pm 0.15 \mu \mathrm{g} / \mathrm{kg}$ diet. The diets were analyzed for contamination by other mycotoxins (ochratoxin A, fumonisins, deoxynivalenol, zearalenone); the levels found were under the EU limits for pigs.

\subsection{Determination of Total Antioxidant Status}

A total antioxidant capacity (TAC) assay has already been described by Marin et al. [39]. The method consists of the measurement of the absorption of 2,20-azinobis-[3-ethylbenzothiazoline-6-sulfonic acid cation (ABTS+)] in samples of mesenteric lymph nodes, and the results are expressed as mmol TEAC (trolox equivalent antioxidant capacity)/g tissue.

\subsection{TBARS Assessment}

Thiobarbituric acid reactive substances (TBARS) were measured in samples of frozen mesenteric lymph nodes, as already described [39]. The results are expressed as nmol/mg protein.

\subsection{Enzyme Activity Assessment}

The activities of superoxide dismutase (SOD), catalase (CAT), and glutathione peroxidase (GPx) were measured using Cayman kits (Cayman Chemical, Ann Arbor, MI, USA), according to the instructions provided by the manufacturer [52]. A Tecan microplate reader (SunRise, Vienna, Austria) was used for the measurement of the absorbance.

\subsection{Cytokine Measurement}

Cytokine concentration was assessed in mesenteric lymph nodes, as already described by Marin et al. [39]. Briefly, the homogenates from $1 \mathrm{~g}$ of frozen sample for each animal, in buffer with complete protease inhibitor cocktail, were used for the analyses of cytokine content by ELISA. Bradford assay was used for the analyses of total protein content. Monoclonal antiporcine antibodies for IL-1beta, IL-6, IL-8, and TNF alpha were used as capture antibodies, and biotinylated antiporcine IL-1beta, IL-6, IL-8, and TNF alpha were used as secondary antibodies (R\&D Systems; Minneapolis, MN, USA). Results were presented as micrograms of cytokine/g tissue. 


\subsection{Extraction of Total RNA and cDNA Synthesis}

RNA extraction followed by cDNA synthesis was carried out as described by Pistol et al. [53]. Briefly, a Qiagen RNeasy midi kit (QIAGEN GmbH, Hilden, Germany) was used for the extraction of total RNA from frozen mesenteric lymph node samples and then treated with ribonuclease inhibitors and purified on columns of silica gel. Concentration and quality were analyzed using a Nanodrop ND-1000 (Thermo Fischer Scientific, Waltham, MA, USA), and integrity was analyzed using agarose gel electrophoresis. Then, $100 \mathrm{ng}$ of total RNA samples were used for the generation of cDNA using an M-MuLV Reverse Transcriptase Kit (Thermo Fischer Scientific, Waltham, MA, USA). A GeneQuerry ${ }^{\mathrm{TM}}$ Pig cDNA Evaluation Kit (ScienCell, Carlsbad, CA, USA) was used for the assessment of the absence of contamination with genomic DNA and the successful reverse transcription of tRNA to cDNA and cDNA quality.

\subsection{Detection of Inflammatory and Signalling Gene Expression by $q P C R$ Array}

Real-time PCR was used to evaluate the expression of transcription factors: c-Jun, nuclear factor NF-kappa-B p65 (NF-kB p65), and signal transducer and activator of transcription 3 (STAT3); MAP kinases: extracellular signal-regulated kinase (ERK 1), c-Jun N-terminal kinase (JNK 1, JNK2) and p38, and metalloproteinase MMP2; cytokines: tumor necrosis factor (TNF alpha), interleukin beta (IL-1 beta), interferon gamma (IFN gamma), interleukin 8 (IL-8), and interleukin 18 (IL-18), as already described by Marin et al. [40]. The sequence of gene-specific primer pairs and the conditions used for the reactions have already been published in our previous papers [54-56]. Duplicates were performed for each gene, and melting curves were used for the confirmation of the formation of single PCR products. For all primers, negative controls were used, consisting of qPCR mix except for cDNA. Two reference genes-beta-actin (ACTB) and hypoxanthine-guanine phosphoribosyl-transferase (HGPRT) - were used for the relative quantification of gene expression changes, and the results were expressed as fold change, as compared with the control group ( $\mathrm{Fc})$, using the $2^{(-\Delta \Delta C T)}$ method [57].

\subsection{Western Blot Analysis}

The protein expression level of three proteins involved in cell signaling-MAPK-p38, NF-kB phosphorylated form and Nrf2 (Nuclear factor erythroid 2-related factor 2)-were measured by Western blot. Cytoplasmic and nuclear fractions of tissue lysates were obtained using the protocol recommended by Thermo Fisher Scientific (Rockford, IL, USA NE-PER) for Nuclear and Cytoplasmic Extraction Reagent Kits, as described by Pistol et al. [53]. After assessment of protein concentration (Pierce BCA Protein Assay Kit, Thermo Fischer Scientific, Waltham, MA, USA), lymph nodes lysates, undiluted (cytoplasmic lysates) or $\frac{1}{2}$ diluted (nuclear lysates), were separated on a $10 \%$ SDS-PAGE and transferred onto a $0.45-\mu \mathrm{m}$ nitrocellulose membrane. After being blocked overnight with $5 \%$ nonfat dry milk, the membrane was incubated after washing with primary antibodies from Cell Signaling Technology (Beverly, MA, USA) for phospho-MAPK-p38 (rabbit antiporcine phospho-MAPK-p38), phospho-NF-kB/p65 (rabbit antiporcine phospho-NF-kB p65), $\beta$-actin (rabbit antiporcine $\beta$-actin), and Nrf2 (rabbit polyclonal antibody; Abbexa, Cambridge, UK) for $2 \mathrm{~h}$ at room temperature. Then, the membranes were incubated with a horseradish-peroxidase-conjugated antirabbit IgG antibody for $1 \mathrm{~h}$ (Cell Signaling Technology, Danvers, MA, USA). The immunoreactivity was assessed by using Clarity Western ECL Substrate (Bio-Rad, Hercules, CA, USA). A MicroChemi Imager (DNR Bio-Imaging Systems LTD, Neve Yamin, Israel) was used for developing immunoblotting images, and GelQuant software (DNR Bio-Imaging Systems LTD, Neve Yamin, Israel) was used for the evaluation of the level of protein expression. The results represent the ratio between the expression level of the protein of interest (p38 MAPK, NF-kB/p65, Nrf2) and $\beta$-actin. 


\subsection{Statistical Analyses}

The differences for all the investigated parameters were analyzed using one-way ANOVA tests, followed by Fisher's PSLD test.

Author Contributions: Conceptualization, D.E.M. and I.T.; assessment of oxidative stress parameters, M.L.P. and M.I.D., Western blot analyses, C.A.A., C.V.B. and G.C.P.; qPCR analyses C.V.B. and G.C.P., data analysis and statistics, D.E.M.; writing-original draft preparation, D.E.M.; writing-review and editing, I.T.; funding acquisition, D.E.M. and I.T. All authors have read and agreed to the published version of the manuscript.

Funding: This research was funded by National Research Projects PN III-8PCCDI, granted by the Romanian Ministry of Education and Research.

Conflicts of Interest: The authors declare no conflict of interest.

\section{References}

1. Grenier, B.; Applegate, T.J. Modulation of intestinal functions following mycotoxin ingestion: Meta-analysis of published experiments in animals. Toxins 2013, 5, 396-430. [CrossRef]

2. Sarma, U.P.; Bhetaria, P.J.; Devi, P.; Varma, A. Aflatoxins: Implications on health. Indian J. Clin. Biochem. 2017, 32, 124-133. [CrossRef] [PubMed]

3. Marchese, S.; Polo, A.; Ariano, A.; Velotto, S.; Costantini, S.; Severino, L. Aflatoxin b1 and m1: Biological properties and their involvement in cancer development. Toxins 2018, 10, 214. [CrossRef] [PubMed]

4. Taniwaki, M.H.; Pitt, J.I.; Magan, N. Aspergillus species and mycotoxins: Occurrence and importance in major food commodities. Curr. Opin. Food Sci. 2018, 23, 38-43. [CrossRef]

5. Liew, W.-P.-P.; Mohd-Redzwan, S. Mycotoxin: Its impact on gut health and microbiota. Front. Cell Infect. Microbiol. 2018, 8, 60. [CrossRef]

6. Yin, H.; Jiang, M.; Peng, X.; Cui, H.; Zhou, Y.; He, M.; Zuo, Z.; Ouyang, P.; Fan, J.; Fang, J. The molecular mechanism of g2m cell cycle arrest induced by afb1 in the jejunum. Oncotarget 2016, 7, 35592-35606. [CrossRef]

7. Peng, X.; Chen, K.; Chen, J.; Fang, J.; Cui, H.; Zuo, Z.; Deng, J.; Chen, Z.; Geng, Y.; Lai, W. Aflatoxin b1 affects apoptosis and expression of bax, bcl-2, and caspase- 3 in thymus and bursa of fabricius in broiler chickens. Environ. Toxicol. 2016, 31, 1113-1120. [CrossRef]

8. Yang, X.; Liu, L.; Chen, J.; Xiao, A. Response of intestinal bacterial flora to the long-term feeding of aflatoxin b1 (afb1) in mice. Toxins 2017, 9, 317. [CrossRef]

9. Akbari, P.; Braber, S.; Varasteh, S.; Alizadeh, A.; Garssen, J.; Fink-Gremmels, J. The intestinal barrier as an emerging target in the toxicological assessment of mycotoxins. Arch. Toxicol. 2017, 91, 1007-1029. [CrossRef]

10. Romero, A.; Ares, I.; Ramos, E.; Castellano, V.; Martínez, M.; Martínez-Larrañaga, M.R.; Anadón, A.; Martínez, M.A. Mycotoxins modify the barrier function of caco-2 cells through differential gene expression of specific claudin isoforms: Protective effect of illite mineral clay. Toxicology 2016, 353-354, 21-33. [CrossRef]

11. Kohan, A.B.; Yoder, S.M.; Tso, P. Using the lymphatics to study nutrient absorption and the secretion of gastrointestinal hormones. Physiol. Behav. 2011, 105, 82-88. [CrossRef] [PubMed]

12. Alexander, J.S.; Ganta, V.C.; Jordan, P.A.; Witte, M.H. Gastrointestinal lymphatics in health and disease. Pathophysiology 2010, 17, 315-335. [CrossRef]

13. Worbs, T.; Bode, U.; Yan, S.; Hoffmann, M.W.; Hintzen, G.; Bernhardt, G.; Förster, R.; Pabst, O. Oral tolerance originates in the intestinal immune system and relies on antigen carriage by dendritic cells. J. Exp. Med. 2006, 203, 519-527. [CrossRef] [PubMed]

14. Pierron, A.; Alassane-Kpembi, I.; Oswald, I.P. Impact of mycotoxin on immune response and consequences for pig health. Anim. Nutr. 2016, 2, 63-68. [CrossRef] [PubMed]

15. Meissonnier, G.; Pinton, P.; Laffitte, J.; Cossalter, A.-M.; Gong, Y.Y.; Wild, C.; Bertin, G.; Galtier, P.; Oswald, I. Immunotoxicity of aflatoxin b1: Impairment of the cell-mediated response to vaccine antigen and modulation of cytokine expression. Toxicol. Appl. Pharmacol. 2008, 231, 142-149. [CrossRef] [PubMed]

16. Turcu, R.; Margareta, O.; Criste, R.D.; Mariana, R.; Tatiana, P.; Șoica, C.; Drăgotoiu, D. The effect of using grape seeds meal as natural antioxidant in broiler diets enriched in fatty acids, on meat quality. J. Hyg. Eng. Des. 2019, 35, 14-20. 
17. Chedea, V.S.; Palade, L.M.; Marin, D.E.; Pelmus, R.S.; Habeanu, M.; Rotar, M.C.; Gras, M.A.; Pistol, G.C.; Taranu, I. Intestinal absorption and antioxidant activity of grape pomace polyphenols. Nutrients 2018, 10, 588. [CrossRef]

18. Veskoukis, A.S.; Kyparos, A.; Nikolaidis, M.G.; Stagos, D.; Aligiannis, N.; Halabalaki, M.; Chronis, K.; Goutzourelas, N.; Skaltsounis, L.; Kouretas, D. The antioxidant effects of a polyphenol-rich grape pomace extract in vitro do not correspond in vivo using exercise as an oxidant stimulus. Oxid. Med. Cell Longev. 2012, 2012, 185867. [CrossRef]

19. Taranu, I.; Habeanu, M.; Gras, M.A.; Pistol, G.C.; Lefter, N.; Palade, M.; Ropota, M.; Sanda Chedea, V.; Marin, D.E. Assessment of the effect of grape seed cake inclusion in the diet of healthy fattening-finishing pigs. J. Anim. Physiol. Anim. Nutr. 2018, 102, e30-e42. [CrossRef]

20. Abdel-Wahhab, M.A.; El-Nekeety, A.A.; Hathout, A.S.; Salman, A.S.; Abdel-Aziem, S.H.; Sabry, B.A.; Hassan, N.S.; Abdel-Aziz, M.S.; Aly, S.E.; Jaswir, I. Bioactive compounds from aspergillus niger extract enhance the antioxidant activity and prevent the genotoxicity in aflatoxin b1-treated rats. Toxicon 2020, 181, 57-68. [CrossRef]

21. Oskoueian, E.; Abdullah, N.; Zulkifli, I.; Ebrahimi, M.; Karimi, E.; Goh, Y.M.; Oskoueian, A.; Shakeri, M. Cytoprotective effect of palm kernel cake phenolics against aflatoxin b1-induced cell damage and its underlying mechanism of action. BMC Complement. Altern. Med. 2015, 15, 392. [CrossRef] [PubMed]

22. Lu, H.; Liu, F.; Zhu, Q.; Zhang, M.; Li, T.; Chen, J.; Huang, Y.; Wang, X.; Sheng, J. Aflatoxin b1 can be complexed with oxidised tea polyphenols and the absorption of the complexed aflatoxin b1 is inhibited in rats. J. Sci. Food Agric. 2017, 97, 1910-1915. [CrossRef] [PubMed]

23. Ali Rajput, S.; Sun, L.; Zhang, N.; Mohamed Khalil, M.; Gao, X.; Ling, Z.; Zhu, L.; Khan, F.A.; Zhang, J.; Qi, D. Ameliorative effects of grape seed proanthocyanidin extract on growth performance, immune function, antioxidant capacity, biochemical constituents, liver histopathology and aflatoxin residues in broilers exposed to aflatoxin $\mathrm{b}_{1}$. Toxins 2017, 9, 371. [CrossRef] [PubMed]

24. Taranu, I.; Hermenean, A.; Bulgaru, C.; Pistol, G.C.; Ciceu, A.; Grosu, I.A.; Marin, D.E. Diet containing grape seed meal by-product counteracts afb1 toxicity in liver of pig after weaning. Ecotoxicol. Environ. Saf. 2020, 203, 110899. [CrossRef]

25. Grosu, I.A.; Pistol, G.C.; Taranu, I.; Marin, D.E. The impact of dietary grape seed meal on healthy and aflatoxin b1 afflicted microbiota of pigs after weaning. Toxins 2019, 11, 25. [CrossRef]

26. Whitlow, L. Evaluation of mycotoxin binders. In Proceedings of the 4th Mid-Atlantic Nutrition Conference, Timonium, MD, USA, 29-30 March 2006.

27. Avantaggiato, G.; Solfrizzo, M.; Visconti, A. Recent advances on the use of adsorbent materials for detoxification of fusarium mycotoxins. Food Addit. Contam. 2005, 22, 379-388. [CrossRef]

28. Sabater-Vilar, M.; Malekinejad, H.; Selman, M.H.; van der Doelen, M.A.; Fink-Gremmels, J. In vitro assessment of adsorbents aiming to prevent deoxynivalenol and zearalenone mycotoxicoses. Mycopathologia 2007, 163, 81-90. [CrossRef]

29. Bočarov-Stančić, A.; Adamović, M.; Salma, N.; Bodroža-Solarov, M.; Vučković-Đisalov, J.; Pantić, V. In vitro efficacy of mycotoxins adsorption by natural mineral adsorbents. Biotechnol. Anim. Husb. 2011, 27, 1241-1251. [CrossRef]

30. Huwig, A.; Freimund, S.; Käppeli, O.; Dutler, H. Mycotoxin detoxication of animal feed by different adsorbents. Toxicol. Lett. 2001, 122, 179-188. [CrossRef]

31. Di Gregorio, M.C.; Neeff, D.V.D.; Jager, A.V.; Corassin, C.H.; Carão, Á.C.D.P.; Albuquerque, R.D.; Azevedo, A.C.D.; Oliveira, C.A.F. Mineral adsorbents for prevention of mycotoxins in animal feeds. Toxin Rev. 2014, 33, 125-135. [CrossRef]

32. Teixeira, A.; Baenas, N.; Dominguez-Perles, R.; Barros, A.; Rosa, E.; Moreno, D.A.; Garcia-Viguera, C. Natural bioactive compounds from winery by-products as health promoters: A review. Int. J. Mol. Sci. 2014, 15, 15638-15678. [CrossRef] [PubMed]

33. Tang, G.-Y.; Zhao, C.-N.; Liu, Q.; Feng, X.-L.; Xu, X.-Y.; Cao, S.-Y.; Meng, X.; Li, S.; Gan, R.-Y.; Li, H.-B. Potential of grape wastes as a natural source of bioactive compounds. Molecules 2018, 23, 2598. [CrossRef] [PubMed]

34. Chedea, V.S.; Pelmus, R.S.; Lazar, C.; Pistol, G.C.; Calin, L.G.; Toma, S.M.; Dragomir, C.; Taranu, I. Effects of a diet containing dried grape pomace on blood metabolites and milk composition of dairy cows. J. Sci. Food Agric. 2017, 97, 2516-2523. [CrossRef] [PubMed] 
35. Avantaggiato, G.; Greco, D.; Damascelli, A.; Solfrizzo, M.; Visconti, A. Assessment of multi-mycotoxin adsorption efficacy of grape pomace. J. Agric. Food Chem. 2014, 62, 497-507. [CrossRef] [PubMed]

36. Gambacorta, L.; Pinton, P.; Avantaggiato, G.; Oswald, I.P.; Solfrizzo, M. Grape pomace, an agricultural byproduct reducing mycotoxin absorption: In vivo assessment in pig using urinary biomarkers. J. Agric. Food Chem. 2016, 64, 6762-6771. [CrossRef] [PubMed]

37. Marin, D.E.; Taranu, I. Overview on aflatoxins and oxidative stress. Toxin Rev. 2012, 31, 32-43. [CrossRef]

38. Hussain, T.; Tan, B.; Yin, Y.; Blachier, F.; Tossou, M.C.B.; Rahu, N. Oxidative stress and inflammation: What polyphenols can do for us? Oxid. Med. Cell Longev. 2016, 2016, 7432797. [CrossRef]

39. Marin, D.E.; Pistol, G.C.; Gras, M.; Palade, M.; Taranu, I. A comparison between the effects of ochratoxin a and aristolochic acid on the inflammation and oxidative stress in the liver and kidney of weanling piglets. Naunyn-Schmiedeberg's Arch. Pharmacol. 2018, 391, 1147-1156. [CrossRef]

40. Marin, D.E.; Pistol, G.C.; Gras, M.A.; Palade, M.L.; Taranu, I. Comparative effect of ochratoxin a on inflammation and oxidative stress parameters in gut and kidney of piglets. Regul. Toxicol. Pharmacol. RTP 2017, 89, 224-231. [CrossRef]

41. Yunus, A.W.; Razzazi-Fazeli, E.; Bohm, J. Aflatoxin b(1) in affecting broiler's performance, immunity, and gastrointestinal tract: A review of history and contemporary issues. Toxins 2011, 3, 566-590. [CrossRef]

42. Benkerroum, N. Chronic and acute toxicities of aflatoxins: Mechanisms of action. Int. J. Environ. Res. Public Health 2020, 17, 423. [CrossRef]

43. Mehrzad, J.; Malvandi, A.M.; Alipour, M.; Hosseinkhani, S. Environmentally relevant level of aflatoxin b(1) elicits toxic pro-inflammatory response in murine cns-derived cells. Toxicol. Lett. 2017, 279, 96-106. [CrossRef]

44. Sangiovanni, E.; Dell'Agli, M. Special issue: Anti-inflammatory activity of plant polyphenols. Biomedicines 2020, 8, 64. [CrossRef]

45. Calder, P.C. Immunoregulatory and anti-inflammatory effects of n-3 polyunsaturated fatty acids. Braz. J. Med. Biol. Res. 1998, 31, 467-490. [CrossRef] [PubMed]

46. Holanda, D.M.; Kim, S.W. Efficacy of mycotoxin detoxifiers on health and growth of newly-weaned pigs under chronic dietary challenge of deoxynivalenol. Toxins 2020, 12, 311. [CrossRef] [PubMed]

47. Van Le Thanh, B.; Lessard, M.; Chorfi, Y.; Guay, F. The efficacy of anti-mycotoxin feed additives in preventing the adverse effects of wheat naturally contaminated with fusarium mycotoxins on performance, intestinal barrier function and nutrient digestibility and retention in weanling pigs. Can. J. Anim. Sci. 2015, 95, 197-209. [CrossRef]

48. Solis-Cruz, B.; Hernandez-Patlan, D.; Petrone, V.M.; Pontin, K.P.; Latorre, J.D.; Beyssac, E.; Hernandez-Velasco, X.; Merino-Guzman, R.; Owens, C.; Hargis, B.M.; et al. Evaluation of cellulosic polymers and curcumin to reduce aflatoxin b1 toxic effects on performance, biochemical, and immunological parameters of broiler chickens. Toxins 2019, 11, 121. [CrossRef]

49. Xu, F.; Wang, P.; Yao, Q.; Shao, B.; Yu, H.; Yu, K.; Li, Y. Lycopene alleviates afb(1)-induced immunosuppression by inhibiting oxidative stress and apoptosis in the spleen of mice. Food Funct. 2019, 10, 3868-3879. [CrossRef]

50. Taranu, I.; Marin, D.E.; Palade, M.; Pistol, G.C.; Chedea, V.S.; Gras, M.A.; Rotar, C. Assessment of the efficacy of a grape seed waste in counteracting the changes induced by aflatoxin b1 contaminated diet on performance, plasma, liver and intestinal tissues of pigs after weaning. Toxicon Off. J. Int. Soc. Toxinol. 2019, 162, 24-31. [CrossRef]

51. Taranu, I.; Gras, M.; Pistol, G.C.; Motiu, M.; Marin, D.E.; Lefter, N.; Ropota, M.; Habeanu, M. Omega-3 pufa rich camelina oil by-products improve the systemic metabolism and spleen cell functions in fattening pigs. PLoS ONE 2014, 9, e110186. [CrossRef]

52. Palade, L.M.; Habeanu, M.; Marin, D.E.; Chedea, V.S.; Pistol, G.C.; Grosu, I.A.; Gheorghe, A.; Ropota, M.; Taranu, I. Effect of dietary hemp seed on oxidative status in sows during late gestation and lactation and their offspring. Anim. Open Access J. 2019, 9, 194. [CrossRef]

53. Pistol, G.C.; Braicu, C.; Motiu, M.; Gras, M.A.; Marin, D.E.; Stancu, M.; Calin, L.; Israel-Roming, F.; Berindan-Neagoe, I.; Taranu, I. Zearalenone mycotoxin affects immune mediators, mapk signalling molecules, nuclear receptors and genome-wide gene expression in pig spleen. PLoS ONE 2015, 10, e0127503. [CrossRef] [PubMed]

54. Marin, D.E.; Pistol, G.C.; Neagoe, I.V.; Calin, L.; Taranu, I. Effects of zearalenone on oxidative stress and inflammation in weanling piglets. Food Chem. Toxicol. 2013, 58, 408-415. [CrossRef] [PubMed] 
55. Pistol, G.C.; Gras, M.A.; Marin, D.E.; Israel-Roming, F.; Stancu, M.; Taranu, I. Natural feed contaminant zearalenone decreases the expressions of important pro- and anti-inflammatory mediators and mitogen-activated protein kinase/nf-kappab signalling molecules in pigs. Br. J. Nutr. 2014, 111, $452-464$. [CrossRef] [PubMed]

56. Marin, D.E.; Motiu, M.; Pistol, G.C.; Gras, M.A.; Israel-Roming, F.; Calin, L.; Stancu, M.; Taranu, I. Diet contaminated with ochratoxin a at the highest level allowed by eu recommendation disturbs liver metabolism in weaned piglets. World Mycotoxin J. 2016, 9, 587-596. [CrossRef]

57. Livak, K.J.; Schmittgen, T.D. Analysis of relative gene expression data using real-time quantitative pcr and the 2(-delta delta $\mathrm{c}(\mathrm{t}))$ method. Methods 2001, 25, 402-408. [CrossRef]

Publisher's Note: MDPI stays neutral with regard to jurisdictional claims in published maps and institutional affiliations.

(C) 2020 by the authors. Licensee MDPI, Basel, Switzerland. This article is an open access article distributed under the terms and conditions of the Creative Commons Attribution (CC BY) license (http://creativecommons.org/licenses/by/4.0/). 